\title{
Pancytopenia of Unknown Cause in Adult Patients Admitted to a Tertiary Hospital in Ethiopia: Case series
}

\author{
Esayas Kebede Gudina ${ }^{1 *}$, Hiwot Amare ${ }^{1}$, Kasahun Benti ${ }^{1}$, Shoba Ibrahim ${ }^{1}$, \\ Gashahun Mekonnen ${ }^{1}$
}

OPEN ACCESS

Citation: Esayas Kebede Gudina, Hiwot Amare, Kasahun Benti, Shoba Ibrahim, Gashahun Mekonnen. Pancytopenia of Unknown Cause in Adult patients Admitted to a Tertiary Hospital in Ethiopia: Case series. Ethiop J Health Sci.2017; 28(4): 375.

doi:http://dx.doi.org/10.4314/ejhs.v28i4.3

Received: April 12, 2017

Accepted: October 8, 2017

Published: July 1, 2018

Copyright: (C) 2018 Esayas Kebede Gudina, et al. This is an open access article distributed under the terms of the

Creative Commons Attribution License, which permits unrestricted use, distribution, and reproduction in any medium, provided the original author and source are credited.

Funding: Nil

Competing Interests: The authors declare that this manuscript was approved by all authors in its form and that no competing interest exists.

Affiliation and Correspondence:

${ }^{1}$ Department of Internal Medicine,

Jimma University, Jimma

*Email: esakgd@gmail.com

\section{ABSTRACT}

BACKGROUND: Over the past few years, we have witnessed a dramatic increase in the number of patients presenting with severe pancytopenia to Jimma University Hospital. We now present sociodemographic and clinical characteristics of adult patients admitted with pancytopenia of unknown cause to Jimma University Hospital during the period of March 2015 to June 2016. Complete blood count and other diagnostic tests were done for all patients to uncover underlying causes.

RESULT: Out of 65 cases admitted with pancytopenia during the specified period, 40 were excluded for various reasons. The rest 25 patients were included in this review. The mean age was 32.1 years $(S D=14.9) ; 14$ were younger than 30 years of age. The mean hemoglobin level, white cell count and platelet count were $48.6 \mathrm{~g} / \mathrm{L}$ $(S D=1.9), 1,918 / \mu L \quad(S D=879.8)$ and $36,200 / \mu L \quad(S D=26,131)$ respectively. The major presenting symptoms were generalized malaise and fever. No geographic or seasonal clustering of the cases was seen.

CONCLUSION: The number of cases with pancytopenia of unidentified cause seen at the hospital over the specified period is alarmingly high and deserves great attention. The hematologic alteration in most of the patients was found to be severe with poor clinical outcome. This calls for large scale community based investigation to uncover the root cause of the problem.

KEYWORDS: Aplastic anemia, Pancytopenia, Ethiopia, Jimma

\section{INTRODUCTION}

Pancytopenia is a clinical phenomenon characterized by reduction in the levels of all the three types of blood cells-erythrocytes, leukocytes and platelets. It is not a disease entity on its own but a triad of cytopenias originating from various disease processes (1). Pancytopenia can be due to bone marrow failure, immune mediated destruction of cells or sequestration in peripheral tissues (2). Aplastic anemia, acute leukemias, bone marrow fibrosis, myelodysplastic syndrome and infiltrative diseases mainly due to malignant cells are major causes of bone marrow failure. Hypersplenic state, systemic lupus erythematosus, infections (Tuberculosis, HIV, leishmaniasis and brucellosis) and nutritional deficiency (folate, B12) are non-marrow disorders associated with pancytopenia (2). 
Even though causes of pancytopenia exhibit substantial geographic variations, aplastic anemia is the commonest cause of pancytopenia worldwide $(3,4)$. Aplastic anemia can be inherited or acquired. Acquired aplastic anemia due to drugs, viral infections, various organic compounds and radiation is by far the commonest form (2). However, more than half of the patients with aplastic anemia do not have known causes $(2,5,6)$. Moreover, the pathophysiologic mechanisms of aplastic anemia in some of the known causes like certain drugs and virus is not well known (2).

Chemotherapeutic agents, antibiotics (chloramphenicol, sulphonamides), antiepileptic drugs (Carbamazepine, Phenytoin, Valproic acid), and heavy metals such as gold are known therapeutic agents associated with increased risk of aplastic anemia. Virus such as parvovirus B19, hepatitis viruses (non-A, non-B, non-C, non-G hepatitis) and HIV are known viral causes of aplastic anemia $(2,7)$. Aplastic anemia due to exposure to industrial chemicals, pesticides and insecticides such as benzene hexacholride (lindane) are also well documented (8-11). Pesticide and insecticide related aplastic anemia often affects young and working country-side men in low income settings who have prolonged exposure to these chemicals $(9,12,13)$. However, the contribution of these organic agents is often overlooked and data, from sub-Saharan Africa in particular, remain scarce.

There are no specific signs and symptoms for pancytopenia. Clinical presentations are directly proportional to the severity of the underlying cytopenias as well as the etiology (14). Patients with mild cytopenia are often asymptomatic (15). The majority of symptoms are related to the severe anemia and catabolic state. As a result, the commonest presenting symptom is generalized weakness. Dyspnea, fever and weight loss are also common $(15,16)$. Furthermore, patients with severe thrombocytopenia may present with bleeding diathesis (15).

Management and prognosis of pancytopenia is complex and depends on underlying pathophysiology and patient related factors (15). Supportive management including treatment of the anemia, thrombocytopenia and infections are important life-saving emergency treatments. Pancytopenias due to nutritional deficiencies like megaloblastic anemia are readily treatable and reversible (16). However, the clinical outcome largely depends on the severity of the pancytopenia, age of the patient and the causes. Hence, identifying the underlying etiology is essential for proper management and to improve long term outcome $(17,18)$.

Over the last few years, we have witnessed a dramatic increase in the number of cases presenting with severe pancytopenia admitted to Jimma University Hospital. Many of these patients were young and did not have known causes or identifiable risk factors for pancytopenia. Because of limited diagnostic capacity to uncover underlying causes, the management of these patients remains very challenging for physicians. The objective of this review was to describe the sociodemographic and clinical characteristics of patients admitted to the hospital with pancytopenia of unknown causes during a period of 16 months. The main goal is to provide clinical summary and baseline information to initiate large scale epidemiologic investigation and interventions for the problem.

\section{METHODS}

This case series survey was conducted at Jimma University Hospital in southwest Ethiopia. Data were collected both retrospectively and prospectively. Patients included in the survey were those admitted to medical wards of the hospital between March 2015 and June 2016. For patients admitted between March to December, 2015, data was collected retrospectively. For patients admitted after that, data was collected prospectively.

Cases included in this review were selected based on the following inclusion criteria:

1. Leukopenia, white cell count $<4000$ cell $/ \mu \mathrm{L}$

2. Anemia, hemoglobin $<100 \mathrm{~g} / \mathrm{L}$

3. Thrombocytopenia, platelet count $<100,000$ cells $/ \mu \mathrm{L}$

Patients with known or suspected causes of pancytopenia were excluded from this review. As 
a result, patients with confirmed or possible diagnosis of any of the following were not included:

1. Any hypersplenic state (massive splenomegaly)-e.g., hyperactive malaria splenomegaly, portal hypertension (hepatosplenic schistosomiasis)

2. Megaloblastic anemia

3. Hematologic malignancies (lymphomas and leukemias)

4. Confirmed solid organ malignancy

5. HIV infection

6. Disseminated tuberculosis (miliary tuberculosis)

7. Decompensated chronic liver disease

8. Patients taking any drug known to cause pancytopenia (chemotherapy, sulfonamides, etc.)

9. Severe systemic bacterial infections (e.g., sepsis of any source)

10. Serologically confirmed acute viral hepatitis

11. Severe iron deficiency state

12. Systemic lupus erythematosus and any known autoimmune disorder

13. Visceral leishmaniasis (suspected or confirmed)

Sociodemographic characterises and presenting clinical symptoms were retrieved through patient interview where applicable or chart review in retrospective cases. Patients were thoroughly screened for inclusion and exclusion criteria through history-taking/chart review, physical examination and laboratory parameters. All patients had complete blood count by coulter counter, erythrocyte sedimentation rate (ESR) and liver enzymes. They were also offered rapid HIV test. Peripheral morphology was also done, and other diagnostic investigations were performed to exclude potential causes of anemia/leukopenia/thrombocytopenia. Information collected through interview and physical examination of the patient and all diagnostic tests performed were done only as part of routine patient care. Outcome at leaving hospital was documented for length of hospital stay, discharge status and way of leaving hospital.

\section{RESULTS}

Sociodemographic and clinical characteristics of the cases: During the period of March 2015 to June 2016, 65 patients were admitted to the hospital with diagnosis of pancytopenia. Forty patients were excluded because they either had known underlying causes or were not fully worked-up, or their medical records were lost. The rest 25 patients were included in this review. The mean age of those included was 32.1 years $(\mathrm{SD}=14.9)$ with range of 16 to 72 . Fourteen $(56 \%)$ were younger than 30 years while only two patients were older than 50. Nearly two-third (16) were male patients. Seventeen patients $(68 \%)$ were rural residents; six were from urban/semiurban settings whereas two were from refugee camp in Gambella Region.

Duration of illness before presentation ranged from 2 to 60 days with mean of 23.5 days $(\mathrm{SD}=18.7)$. The major presenting symptoms were malaise and easy fatigability reported in all of the patients. Fever and vomiting were the other common symptoms reported in 14 and 7 patients respectively (Table 1 ).

Table 1: Presenting clinical symptoms of patients treated for pancytopenia of unknown cause at Jimma University Hospital.

\begin{tabular}{lrr}
\hline Symptoms & Number & Percent \\
\hline Generalized fatigability & 25 & 100 \\
Fever & 14 & 56 \\
Vomiting & 7 & 28 \\
Edema & 6 & 24 \\
Headache & 4 & 16 \\
Decreased appetite & 3 & 12 \\
Epistaxis & 3 & 12 \\
Jaundice & 1 & 4 \\
\hline
\end{tabular}


With regard to comorbidities identified, two patients were on treatment for major depressive disorder with tricyclic antidepressant; one patient had established chronic renal insufficiency and another had chronic viral hepatitis (positive serology only). One patient was pregnant. None of the patients were using any illicit drug or herbal treatment prior to their illnesses.

On examination, 13 patients $(52 \%)$ had objectively detected fever on arrival and three presented with low blood pressure. Hepatomegaly, spleanomegaly, and axillay lymphadenopathy were detected in 5, 4, and 2 patients respectively. Jaundice and petechial rash were detected in one patient each.

All of the patients had peripheral complete blood count using Coulter counter. The mean white cell count was $1,918 / \mu \mathrm{L}(\mathrm{SD}=879.8)$ with range of 500 to $3,700 / \mu \mathrm{L}$. All of the patients had absolute neutrophil count of less than $1,500 / \mu \mathrm{L}$; seven had counts less than $500 / \mu \mathrm{L}$. The mean hemoglobin was $48.6 \mathrm{~g} / \mathrm{L} \quad(\mathrm{SD}=1.9)$ ranging between 17 and $93 \mathrm{~g} / \mathrm{L}$. Twenty-three of the patients $(92 \%)$ had severe anemia (hemoglobin < $80 \mathrm{~g} / \mathrm{L}$ ). The mean platelet count was $36,200 / \mu \mathrm{L}$ $(\mathrm{SD}=26,131)$ with range of 2,000 to 93,000 . Eighteen patients had count less than $50,000 / \mu \mathrm{L}$ and eight had count less than $20,000 / \mu \mathrm{L}$.

Peripheral morphology was evaluated for all of the patients. No morphologic abnormality of nucleated cells was noted. Tear drops and fragmented RBCs were reported in few patients. The mean cell volume was $97.9(\mathrm{SD}=11)$ with range of 83.5 to $118.4 \mathrm{fl}$; nine patients had mild macrocytosis.

Erythrocyte sedimentation rate (ESR) was the only acute phase reactant tested in the patients. Most of them had a very high rate with mean of $108.1 \mathrm{~mm}$ in the first hour ( $\mathrm{SD}=46.4$ ) and range of 40 to $165 \mathrm{~mm}$ in the first hour.
Liver enzymes aspartate aminotransferase (AST) and alanine aminotransferase (ALT) were assessed in all of the patients. Eleven patients had increment in AST (from slight elevation to 17 fold increment from normal upper limit of the laboratory in two patients). Six patients had raised ALP from mild elevation to about 24 fold of normal upper limit. Five patients had hyperbilirubinemia. Serum protein level was reported for four patients only; all had lower than normal values. All the three patients who had coagulation assay also had elevated prothrombin time. However, serologic screening for hepatitis B and hepatitis $\mathrm{C}$ viruses was negative except in one patient who had positive hepatitis B surface antigen who otherwise had normal liver enzyme level.

Ultrasound of abdomen was normal for all except for those with hepatomegaly and splenomegaly. Blood film for hemoparasite was repeatedly performed for all of these patients and was found to be negative in all.

Treatment and outcome of the cases: Twentytwo patients were transfused with one or more units of compatible whole blood, and 17 of them were empirically given intravenous ceftriaxone alone or in combination with other antibiotics. Four patients (16\%) died in the hospital; 12 (48\%) left the hospital without any improvement (five of them were referred for better care and six selfdischarged). Only 9 (36\%) were discharged with some improvement; cell counts in some of these patients significantly improved.

Geographic and seasonal variation of cases: The cases were from Jimma and Illubabor zones of Oromiya Region, Keffa and Sheka zones of South Nations and Nationalities Region, and Gambella Region. More than half of the patients were from Jimma Zone due to proximity to the hospital. However, there is no clustering of cases to specific village, kebele or wereda (Figure 1). 


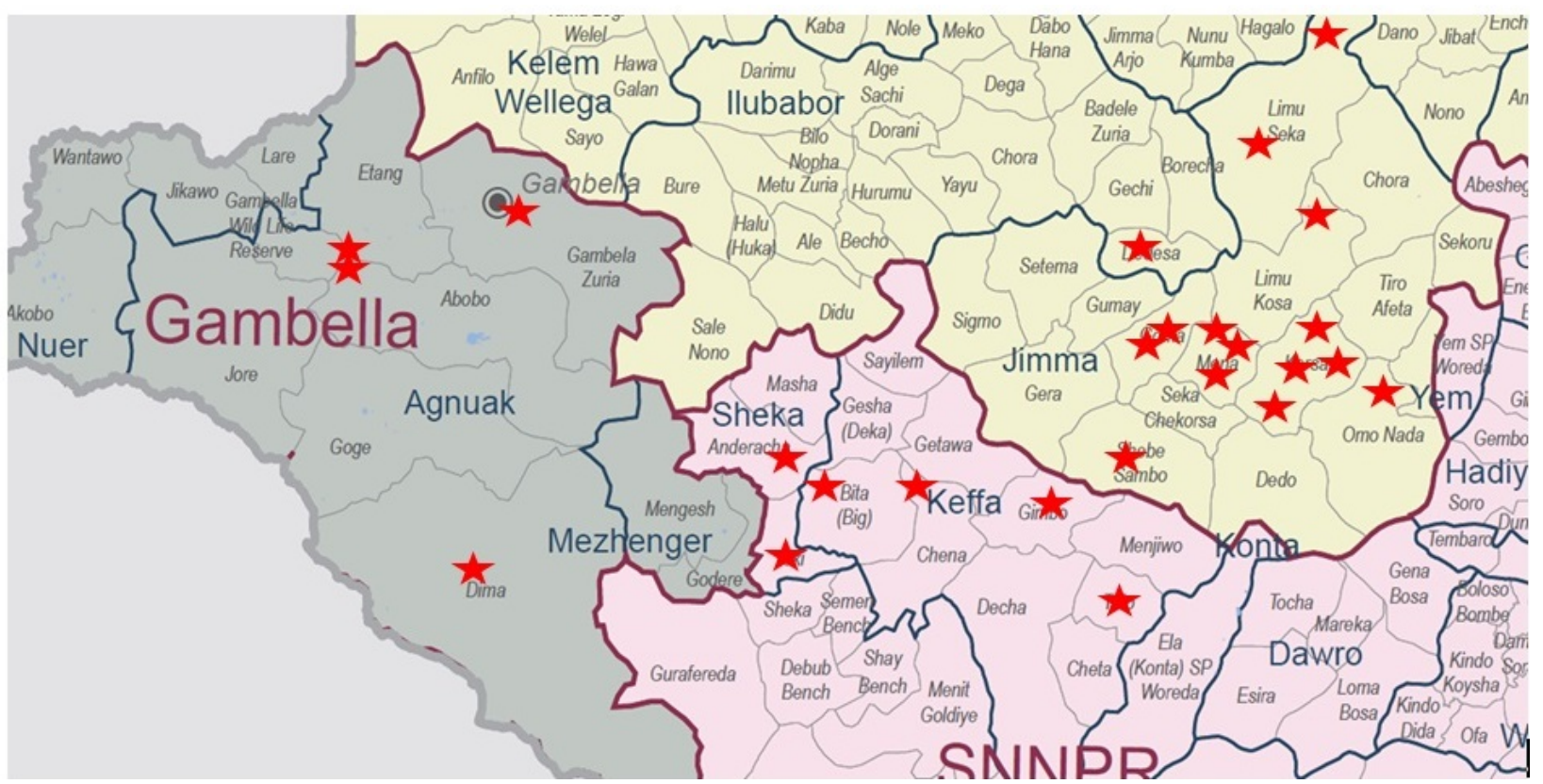

Figure 1: Map of Southwest Ethiopia region illustrating the origin of patients who presented to Jimma University Hospital with pancytopenia

Similarly, there is no clear seasonal variation seen even though the number of cases was found to be higher between December and March (Figure 2). This is most likely due to selection bias; data was collected retrospectively for patients presenting before January 2016 and prospectively after that. As we tried to include only patients with complete data in retrospective cases, the number included here is likely to be an underestimate of real number of cases.

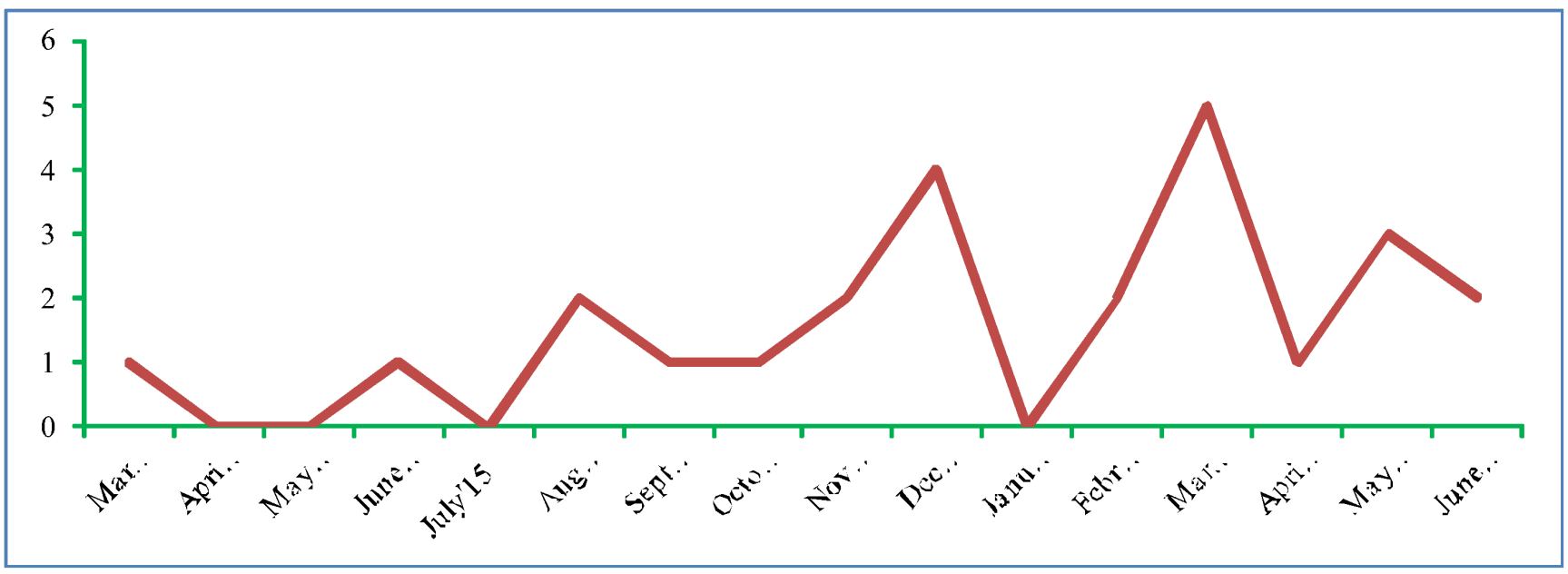

Figure 2: Trends in number of cases admitted with pancytopenia to Jimma University Hospital during 2015-2016

DOI: http://dx.doi.org/10.4314/ejhs.v28i4.3 


\section{DISCUSSION}

Pancytopenia of unknown cause was found to be a common clinical phenomenon at the hospital during the survey period. Most of the patients admitted with the problem were young countryside men presenting with severe pancytopenia. No geographic clustering of the cases was seen with patients presenting from wide areas of southwestern Ethiopia. The underlying causes were not identifiable despite multiple diagnostic tests. The discharge outcome was found to be very poor with only $9(25 \%)$ patients having improvement on leaving hospital; the overall inhospital mortality rate was $16 \%$. This shows that pancytopenia is now a serious and common medical condition in the area. The cause in many patients is unknown and thus the treatment remains obscure.

The reported number of cases during the period is likely to be an underestimate due to lost records. Moreover, it should be noted that none of the patients had bone marrow examination and that some of them might have had known causes. The findings of individual patients in this case review does not imply whether the underlying pathology is bone marrow failure or peripheral destruction. However, due to the fact that patients' presentation was related only to the pancytopenia and the absence of other comorbid condition may reflect primary bone marrow affection. Although the evaluation of these cases falls short of standard investigation for pancytopenia, the absence of known causes with available tests may support diagnosis of aplastic anemia $(2,5,6)$.

Most of our patients were rural residents with poor economic status. Even though this by itself may not imply any underlying cause, environmental factors are likely contributors of the major pathophysiology. Multiple factors may contribute to such environmental factors for aplastic anemia (19), and low socioeconomic status may be a surrogate for environmental factors such as infectious pathogens or toxin exposures (20). The absence of geographic clustering and seasonal variability shows the diverse occurrence of the problem. Thus, investigation for the root cause of this geographically widespread problem should put in to consideration the possibility of commonly used environmental toxins (such as pesticides and insecticides) and infectious conditions such as, but not limited to, vector born viral infections.

Whether the mild hepatocellular alterations as evidenced by elevated liver enzymes and serum bilirubin in some of the patients is an association or coincidental occurrence was not thoroughly investigated in these patients. Nevertheless, hepatitis associated aplastic anemia (21) and environmental exposure to pesticides and insecticides (22) may explain such clinical scenario. However, because of the limitations of this review, we are reserved from making any conclusion from these associations.

Only about a third of the patients had a reported clinical and hematologic improvement on discharge. In addition, many patients left the hospital with poor clinical status. Even though the in-hospital mortality rate was only $16 \%$, it is possible to extrapolate that the mortality in these cases is likely to be much higher. Most affected patients were young and in the productive age group reflecting a huge clinical and economic consequence of the problem and hence deserves utmost attention from all stakeholders.

In conclusion, the number of cases with pancytopenia of unidentified cause seen at the hospital over the specified period is alarmingly high and of great concern. Even at the time of writing of this report, there were still cases with similar clinical presentations. The hematologic alteration in most of the patients was found to be very grave with severe clinical conditions. It is thus mandatory for all stakeholders (the hospital, local health offices, regional health bureau and Federal Ministry of Health) to screen all such cases for possible underlying etiologies. Large scale investigation is essential to reach to the culprit cause and take emergent public health intervention.

Our review of this case series has multiple limitations worth mentioning here. Only patients with complete medical records were included in this survey. Moreover, only patients admitted to the hospital were included leaving for the 
possibility of significant selection bias. Additionally, bone marrow examination was not done for all of them, and hence, the real pathophysiology cause of pancytopenia could not be established. Absence of toxicological and virologic screening for possible underlying causes was additional limitation of this survey.

\section{REFERENCES}

1. Weinzierl EP, Arber DA. Bone marrow evaluation in new-onset pancytopenia. Hum Pathol. 2013;44(6):1154-64.

2. Weinzierl EP, Arber DA. The differential diagnosis and bone marrow evaluation of new-onset pancytopenia. Am J Clin Pathol. 2013;139(1):9-29.

3. Jha A, Sayami G, Adhikari RC, Panta AD, Jha $\mathrm{R}$. Bone marrow examination in cases of pancytopenia. JNMA J Nepal Med Assoc. 2008;47(169):12-7.

4. Biswajit H, Pratim PP, Kumar ST, Shilpi S, Krishna GB, Aditi A. Aplastic anemia: a common hematological abnormality among peripheral pancytopenia. $N \mathrm{Am} J \mathrm{Med} S \mathrm{Sci}$. 2012;4(9):384-8.

5. Storb R. Aplastic anemia. J Intraven Nurs. 1997;20(6):317-22.

6. Keohane EM. Acquired aplastic anemia. Clin Lab Sci. 2004;17(3):165-71.

7. Young NS. Acquired aplastic anemia. Ann Intern Med. 2002;136(7):534-46.

8. Zhang J, Yang T. [Meta-analysis of association between organophosphorus pesticides and aplastic anemia]. Zhonghua Liu Xing Bing Xue Za Zhi. 2015;36(9):1005-9.

9. Issaragrisil S, Chansung K, Kaufman DW, Sirijirachai J, Thamprasit T, Young NS. Aplastic anemia in rural Thailand: its association with grain farming and agricultural pesticide exposure. Aplastic Anemia Study Group. Am J Public Health. 1997;87(9):1551-4.

10. Fleming LE, Timmeny W. Aplastic anemia and pesticides. An etiologic association? J Occup Med. 1993;35(11):1106-16.

11. Rugman FP, Cosstick R. Aplastic anaemia associated with organochlorine pesticide: case reports and review of evidence. J Clin Pathol. 1990;43(2):98-101.

12. Prihartono N, Kriebel D, Woskie S, Thetkhathuek A, Sripaung N, Padungtod C, et al. Risk of aplastic anemia and pesticide and other chemical exposures. Asia Pac J Public Health. 2011;23(3):369-77.

13. Taj M, Shah T, Aslam SK, Zaheer S, Nawab F, Shaheen $\mathrm{S}$, et al. Environmental determinants of

DOI: http://dx.doi.org/10.4314/ejhs.v28i4.3 
aplastic anemia in Pakistan: a case-control study. Z Gesundh Wiss. 2016;24(5):453-60.

14. DeZern AE, Guinan EC. Aplastic anemia in adolescents and young adults. Acta Haematol. 2014;132(3-4):331-9.

15. Gayathri BN, Rao KS. Pancytopenia: a clinico hematological study. $J$ Lab Physicians. 2011;3(1):15-20.

16. Premkumar M, Gupta N, Singh T, Velpandian T. Cobalamin and folic Acid status in relation to the etiopathogenesis of pancytopenia in adults at a tertiary care centre in north India. Anemia. 2012;2012:707402.

17. Zeng Y, Katsanis E. The complex pathophysiology of acquired aplastic anaemia. Clin Exp Immunol. 2015;180(3):361-70.

18. Dezern AE, Brodsky RA. Clinical management of aplastic anemia. Expert Rev Hematol. 2011;4(2):221-30.
19. Gordon-Smith EC, Issaragrisil S. Epidemiology of aplastic anaemia. Baillieres Clin Haematol. 1992;5(2):475-91.

20. Issaragrisil $S$, Kaufman DW, Anderson TE, Chansung K, Thamprasit T, Sirijirachai J, et al. An association of aplastic anaemia in Thailand with low socioeconomic status. Aplastic Anemia Study Group. Br J Haematol. 1995;91(1):80-4.

21. Brown KE, Tisdale J, Barrett AJ, Dunbar CE, Young NS. Hepatitis-associated aplastic anemia. N Engl J Med. 1997;336(15):1059-64.

22. Freire C, Koifman RJ, Koifman S. Hematological and hepatic alterations in Brazilian population heavily exposed to organochlorine pesticides. J Toxicol Environ Health A. 2015;78(8):534-48. 